\title{
Intensivierung von enzymkatalysierten Reaktionen
}

\author{
DIRK HOLTMANN ${ }^{1}$, FRANK HOLLMANN ${ }^{2}$ \\ ${ }^{1}$ INSTITUT FÜR BIOVERFAHRENSTECHNIK UND PHARMAZEUTISCHE TECHNOLOGIE, \\ ARBEITSGRUPPE INTENSIVIERUNG VON BIOPROZESSEN, TECHNISCHE HOCHSCHULE \\ MITTELHESSEN, GIESSEN \\ 2 DEPARTMENT OF BIOTECHNOLOGY, DELFT UNIVERSITY OF TECHNOLOGY, DELFT, \\ NIEDERLANDE
}

\section{Process intensification aims at enabling bridging the gap between fundamental research such as identification of new catalysts and reac- tions and their implementation in industrial environments. Especially the field of biocatalysis has seen some tremendous improvements and the development of new tools and approaches to bridge this gap. In this contribution we highlight some recent developments as selected case studies.}

DOI: $10.1007 / \mathrm{s} 12268-021-1540-5$

(C) Die Autoren 2021

In der Enzymtechnik wurden in den letzten Jahren sehr große Erfolge erzielt bei der Identifizierung neuer Enzyme, der Optimierung der Enzyme mittels rationaler und evolutiver Methoden und bei der Etablierung neuer Reaktionen. Häufig ist allerdings die Performanz der resultierenden Prozesse, z. B. hinsichtlich der Produktivitäten, der erreichbaren Produktkonzentrationen, der Stabilität der Enzyme sowie der Skalierbarkeit noch limitiert. Hier können die Methoden der Prozessintensivierung dazu beitragen, technische Lösungen zu entwickeln, in denen die Enzyme ihr volles Synthesepotenzial ausschöpfen können. Allgemeines Ziel der Prozessintensivierung (PI) ist es, durch den Einsatz innovativer Prinzipien im Prozess- und Ausrüstungsdesign bedeutende Vorteile in Bezug auf Prozesseffizienz, Kapital- und Betriebskosten, Qualität, Abfallmengen und Prozesssicherheit $\mathrm{zu}$ erzielen (s. auch BIOspektrum 1/21, S. 94). Dies bedeutet sehr häufig einen Paradigmenwechsel, der zum Einsatz von interdisziplinären Techniken führt. Abbildung 1 zeigt Beispiele für den Einsatz der PI in der Enzymkatalyse - einige der Beispiele werden im Folgenden diskutiert.

\section{Neue Lösungsmittel}

Lebende Zellen bestehen zu ca. 75 Prozent aus Wasser. Diese Tatsache hat dazu beigetragen, dass wir lebende Zellen als wässrige Lösungen von Enzymen und Metaboliten wahrnehmen. Hinzu kommt, dass Wasser als „grünes Lösungsmittel“ weitgehend anerkannt ist (natürlich ist Wasser an sich aus ökologischer Sicht unproblematisch; anders verhält es sich aber mit Wasser, welches durch die Reagenzien biokatalytischer Reaktionen kontaminiert ist). Deshalb ist es nachvollziehbar, dass Enzymreaktionen zumeist in wässrigen Lösungen durchgeführt werden. Für viele (hydrophobe) Reagenzien ist Wasser allerdings ein eher ungeeignetes Lösungsmittel und ermöglicht oft nur millimolare Reagenzkonzentrationen. Dies bedeutet auch, dass typischerweise weniger als zwei Gramm Produkt pro Liter Reaktionslösung gebildet werden. Pro Gramm Produkt entstehen also auch mehr als 500 Gramm Abwasser, die aufgereinigt werden müssen. Aus unserer Sicht verdeutlichen diese Zahlen, dass verdünnte Reaktionslösungen weder ökonomisch sinnvoll noch „grün“ sind.

Nicht-wässrige, sogar lösungsmittelfreie, biokatalytische Verfahrensweisen sind im
Bereich Hydrolase-katalysierter Veresterungen bereits weit verbreitet [1]. Aber auch bei anderen Enzymreaktionen, wie Ketonreduktionen [2, 3] oder Oxyfunktionalisierungen [4], sind wässrige Reaktionsmedien nicht unbedingt nötig. Seit einigen Jahren treten deep eutectic solvents (DES) als umweltfreundlichere Alternativen zu den in Ungnade gefallenen ionic liquids in den Fokus biokatalytischer Reaktionen [5]. Die Zeit wird zeigen, ob sich DES wirklich in vielfältigen Anwendungen durchsetzen werden.

Für einige Reaktionen (wenn beispielsweise ausschließlich wasserlösliche Reagenzien oder diffundierende Ko-Faktoren unumgänglich sind), in denen auf Wasser nicht verzichtet werden kann, haben sich mehrphasige Reaktionskonzepte (two-liquid phase systems oder slurry-to-slurry-Reaktionen) bewährt. Nichtsdestotrotz bleiben noch Herausforderungen hinsichtlich der Biokatalysatorstabilität und Massentransportlimitationen anzugehen.

\section{Kaskadereaktionen}

Der Großteil der heutigen Produkte lässt sich nicht in einfachen Einschrittsynthesen aus geeigneten Ausgangsmaterialien herstellen. Meist bedarf es mehrerer Syntheseschritte, um das Endprodukt zu synthetisieren. Klassischerweise erfolgen die einzelnen Syntheseschritte sequenziell und mit Aufreinigung der Zwischenprodukte. Diese Vorgehensweise ist ökonomisch und ökologisch ineffizient. Die bestehende Infrastruktur wird oft zur Produktaufreinigung und -isolierung statt zur Synthese verwendet. Darüber hinaus ist die Produktisolation zeit- und resourcenintensiv [6]. Daher sind Kaskadenreaktionen, in denen mehrere Einzelschritte innerhalb eines Reaktionsschritts (idealerweise auch gleichzeitig) durchgeführt werden, besonders attraktiv. Aufgrund überlappender Prozessfenster der einzelnen Katalysatoren sind enzymatische Reaktionen prädestiniert für solche Kaskadenreaktionen. So konnten beispielsweise Li und Mitarbeiter zeigen, dass sich Enzymkaskaden für die 


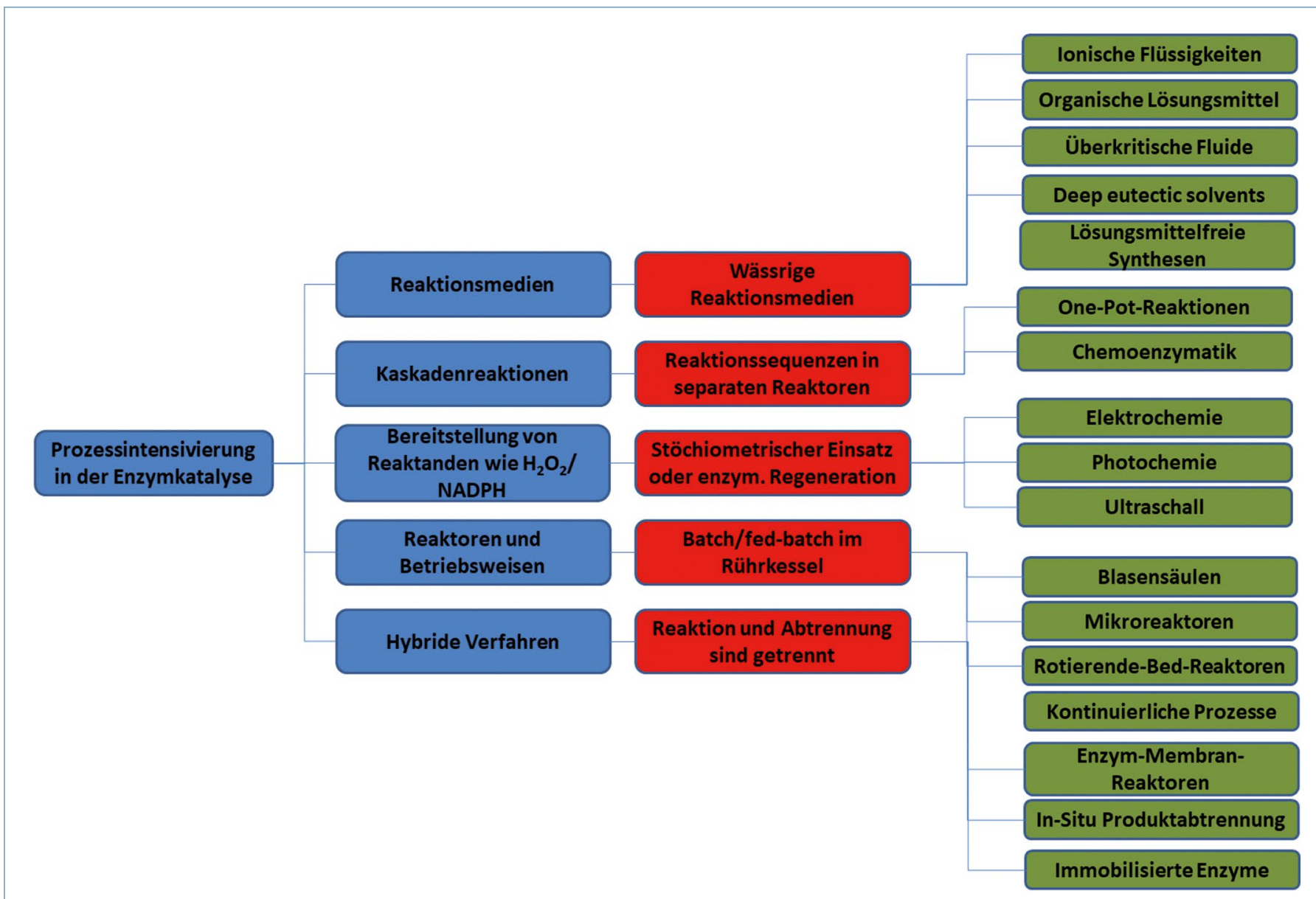

$\Delta$ Abb. 1: Die Prozessintensivierung (PI) in der Enzymkatalyse. blau: in der PI adressiertes Verfahren, rot: Ausgangspunkt eines hypothetischen „Standardprozesses“, grün: Werkzeuge und Methoden im PI.

Synthese einer Vielzahl komplexer Produkte aus einfachen Ausgangsmaterialien eignen [7-9].

\section{Alternative Ko-Faktor- Regenerationskonzepte}

Viele enzymatische Transformationen sind Ko-Faktor-abhängig und bedürfen der kontinuierlichen Regeneration der aktiven KoFaktoren. Viele etablierte, biomimetische Verfahren lehnen sich an natürlich auftretende Regenerationszyklen an und sind somit letztlich glucoseabhängig. Insbesondere bei großvolumigen Produkten ist der stöchiometrische Einsatz von Glucose aus ökonomischer und ethischer Sicht allerdings abzulehnen. Als alternative Energiequellen zur Regeneration biokatalytischer Reaktionen bieten sich (Sonnen-)Licht $[10,11]$ und nachhaltig produzierter Strom an $[12,13]$. Aber auch unkonventionelle Energiequellen - wie Ultraschall [14, 15], Plasmen [16] oder sogar radioaktiver Abfall [17] - könnten Anwendung finden.

\section{Reaktoren und hybride Verfahren}

Klassische Reaktorkonzepte stehen noch weitgehend im Fokus der Biokatalyseforschung. Anscheinend ist der Schritt vom Eppendorf Tube zum Rührreaktor zu offensichtlich; dies gilt auch für viele industrielle Verfahren. Allerdings setzen sich immer mehr (semi-)kontinuierliche Verfahren mit immobilisierten Biokatalysatoren durch. Beispiele hierfür sind der 7-ACA-Prozess, die Glucoseisomerisierung (high fructose corn sirup) und die Synthese von Acrylamid [18]. Solche (semi-)kontinuierlichen Reaktionen bieten sich nicht nur aufgrund ihrer Skalierbarkeit an, sondern sind auch attraktive Lösungen für kinetisch kontrollierte Reaktionen. Der flow chemistry-Ansatz ist eine interessante Weiterentwicklung. So lassen sich beispielsweise Energie- und Massetransportlimitationen im flow-Reaktor leichter überwinden als im klassischen batch-Ansatz.

Blasensäulen eignen sich beispielsweise besonders gut für hochviskose Reaktions- mischungen [19] oder gasförmige Reaktanten $[20,21]$.

\section{Zusammenfassung}

Die synthetische Bandbreite der Biokatalyse wächst kontinuierlich. Damit die industrielle Implementierung mit dieser Entwicklung mithalten kann, ist die Prozessintensivierung unabkömmlich. Auch akademische Forscher sollten hinsichtlich der späteren Implementierung die Prozessintensivierung im Auge behalten. Neuartige Reaktions- und Reaktorkonzepte stehen jedenfalls zur Verfügung und warten nur darauf, eingesetzt $\mathrm{zu}$ werden.

\section{Literatur}

[1] Ansorge-Schumacher MB, Thum 0 (2013) Immobilised lipases in the cosmetics industry. Chem Soc Rev 42: 64756490

[2] de Gonzalo G, Lavandera I, Faber K, Kroutil W (2007) Enzymatic reduction of ketones in "micro-aqueous" media catalyzed by ADH-A from Rhodococcus ruber. Org Lett 9: 2163-2166

[3] Erdmann V, Mackfeld U, Rother D, Jakoblinnert A (2014) Enantioselective, continuous (R)- and (S)-2-butanol synthesis: Achieving high space-time yields with recombinant E. coli 
cells in a micro-aqueous, solvent-free reaction system. J Biotechnol 191: 106-112

[4] Rauch MCR, Tieves F, Paul CE et al. (2019) Peroxygenasecatalysed epoxidation of styrene derivatives in neat reaction media. ChemCatChem 11: 4519-4523

[5] Pätzold M, Siebenhaller S, Kara S et al. (2019) Deep

eutectic solvents as efficient solvents in biocatalysis. Trends Biotechnol 37: 943-959

[6] Schrittwieser JH, Velikogne S, Hall M, Kroutil W (2017) Artificial biocatalytic linear cascades for preparation of organic molecules. Chem Rev 118: 270-348

[7] Wu S, Zhou Y, Wang T et al. (2016) Highly regio- and enantioselective multiple oxy- and amino-functionalizations of alkenes by modular cascade biocatalysis. Nat Commun 7: 11917

[8] Wu SK, Liu J, Li Z (2017) Biocatalytic formal anti-Markovnikov hydroamination and hydration of aryl alkenes. ACS Catal 7: 5225-5233

[9] Wu S, Zhou Y, Seet D, Li Z (2017) Regio- and stereoselective oxidation of styrene derivatives to arylalkanoic acids via one-pot cascade biotransformations. Adv Synth Catal 359: 2132-2141

[10] Zhang W, Fernández-Fueyo E, Ni Y et al. (2018)

Selective aerobic oxidation reactions using a combination of photocatalytic water oxidation and enzymatic oxyfunctionalizations. Nat Catal 1: 55-62

[11] Huijbers M, Zhang W, Hollmann F (2018) Light-driven enzymatic decarboxylation of fatty acids. Angew Chem Int Ed 57: $13648-13651$

[12] Bormann S, van Schie M, De Almeida TP et al. (2019)

$\mathrm{H}_{2} \mathrm{O}_{2}$ production at low overpotentials for electroenzymatic halogenation reactions. ChemSusChem 12: 4759-4763
[13] Getrey L, Krieg T, Hollmann F et al. (2014) Enzymatic halogenation of the phenolic monoterpenes thymol and carvacrol with chloroperoxidase. Green Chem 16: 1104-1108 [14] Yoon J, Kim J, Tieves F et al. (2020) Piezobiocatalysis: ultrasound-driven enzymatic oxyfunctionalization of $\mathrm{C}-\mathrm{H}$ bonds. ACS Catalysis 10: 5236-5242

[15] Dengkui S, Ling Z, Songmei S, Wenzhong W (2018) Oxygen reduction reaction for generating $\mathrm{H}_{2} \mathrm{O}_{2}$ through a Piezo-catalytic process over bismuth oxychloride.

ChemSusChem 11: 527-531

[16] Yayci A, Baraibar AG, Krewing M et al. (2020) Plasmadriven in situ production of hydrogen peroxide for biocatalysis. ChemSusChem 13: 2072-2079

[17] Zhang W, Liu H, van Schie MMCH et al. (2020) Nuclear waste and biocatalysis: a sustainable liaison? ACS Catalysis 10: $14195-14200$

[18] Yamada H, Kobayashi M (1996) Nitrile hydratase and its application to industrial production of acrylamide. Biosci Biotechnol Biochem 60: 1391-1400

[19] Hilterhaus L, Thum O, Liese A (2008) Reactor concept for lipase-catalyzed solvent-free conversion of highly viscous reactants forming two-phase systems. Org Proc Res Dev 12: 618-625

[20] Perz F, Bormann S, Ulber R et al. (2020) Enzymatic oxidation of butane to 2-butanol in a bubble column. ChemCatChem 12: 3666-3669

[21] Sluyter G, Kleber J, Perz F et al. (2020) Fermentative oxidation of butane in bubble column reactors. Bioche Eng J 155: 10
Open Access: Dieser Artikel wird unter der Creative Commons Namensnennuns 4.0 International Lizenz veröffentlicht, welche die Nutzung, Vervielfältigung, erlaubt, sofern Sie den/die ursprünglichen Autor(en) und die Quelle ordnungsgemäß nennen, einen Link zur Creative Commons Lizenz beifügen und angeben, ob Änderungen vorgenommen wurden. Die in diesem Artikel enthaltenen Bilder und sonstiges Drittmaterial unterliegen ebenfalls der genannten Creative Commons Lizenz, sofern sich aus der Abbildungslegende nichts anderes ergibt. Sofern das betreffende Material nicht unter der 列 Weiterverwendungen Rechteinhabers einzuholen. Weitere Details zur Lizenz entnehmen Sie bitte der Lizenzinformation auf http://creativecommons.org/licenses/by/4.0/deed.de.

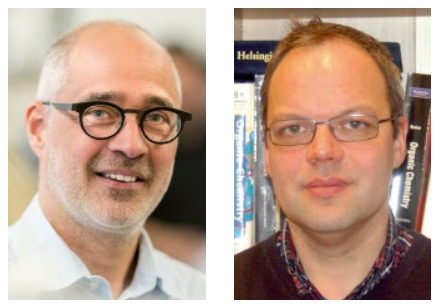

Dirk Holtmann (links) und Frank Hollmann

Korrespondenzadresse:

Prof. Dr. Frank Hollmann

Department of Biotechnology

Delft University of Technology

van der Maasweg 9

NL-2629 HZ Delft

f.hollmann@tudelft.nl

\section{Hier steht eine Anzeige.}

